\title{
Micromechanics of Sea Urchin Spines
}

\author{
Naomi Tsafnat ${ }^{1 *}$, John D. Fitz Gerald ${ }^{2}$, Hai N. Le ${ }^{3}$, Zbigniew H. Stachurski ${ }^{3}$
}

1 School of Mechanical and Manufacturing Engineering, University of New South Wales, Sydney, New South Wales, Australia, 2 Research School of Earth Sciences, Australian National University, Canberra, Australian Capital Territory, Australia, 3 Research School of Engineering, Australian National University, Canberra, Australian Capital Territory, Australia

\begin{abstract}
The endoskeletal structure of the Sea Urchin, Centrostephanus rodgersii, has numerous long spines whose known functions include locomotion, sensing, and protection against predators. These spines have a remarkable internal microstructure and are made of single-crystal calcite. A finite-element model of the spine's unique porous structure, based on micro-computed tomography (microCT) and incorporating anisotropic material properties, was developed to study its response to mechanical loading. Simulations show that high stress concentrations occur at certain points in the spine's architecture; brittle cracking would likely initiate in these regions. These analyses demonstrate that the organization of single-crystal calcite in the unique, intricate morphology of the sea urchin spine results in a strong, stiff and lightweight structure that enhances its strength despite the brittleness of its constituent material.
\end{abstract}

Citation: Tsafnat N, Fitz Gerald JD, Le HN, Stachurski ZH (2012) Micromechanics of Sea Urchin Spines. PLoS ONE 7(9): e44140. doi:10.1371/journal.pone.0044140

Editor: Christof Markus Aegerter, University of Zurich, Switzerland

Received March 8, 2012; Accepted July 30, 2012; Published September 11, 2012

Copyright: (c) 2012 Tsafnat et al. This is an open-access article distributed under the terms of the Creative Commons Attribution License, which permits unrestricted use, distribution, and reproduction in any medium, provided the original author and source are credited.

Funding: The authors have no support or funding to report.

Competing Interests: The authors have declared that no competing interests exist.

*E-mail: n.tsafnat@unsw.edu.au

\section{Introduction}

The endoskeletal structure of the purple-spined Sea Urchin Centrostephanus rodgersii from the New South Wales coast of Australia has on its outside long and numerous spines whose functions include locomotion, sensing, and protection from physical trauma and predators $[1,2]$. The spines protect the spherical test, often by "sacrificing" themselves to absorb energy as they break [1]. In the case of attack by a predator, or impact by an object in surf conditions, the spines can protect the test in two ways. If a predator impacts axially, the spine pierces the object and snaps off, requiring high strength in compression, and brittle fracture in tension or torsion. If an object impacts the spine along its length, it absorbs the energy by brittle fracture in bending. In both cases the energy is absorbed and the load is spread away from the test. Other functions of the spines include locomotion and sensing, which would place significantly less stress on them than impact. For these functions, the spines would need to be axially stiff with enough elasticity to withstand loads in a high energy ocean surge environment.

Sea Urchin spines are made of a single crystal of calcite with the crystallographic c-axis along the spine's length [3]. A monolithic structure comprised of a single crystal of calcite would be very brittle, however Urchin spines are relatively flexible and this has been attributed to a small amount of glycoprotein embedded in the mineral phase that enhances their fracture resistance and increases their elastic limit [3-5]. An intimate mixture of organic and mineral matter in the form of an "oriented array of nanocrystals" has also been used to explain other remarkable properties of urchin-spine biomaterial [6].

In Centrostephanus rodgersii, spines from near the top or sides of the round test are longest and reach up to $10 \mathrm{~cm}$ in length, with diameter varying from approximately $4 \mathrm{~mm}$ at the base reducing to $1 \mathrm{~mm}$ at the tip. Figure $1 \mathrm{~A}$ shows the microstructure characteristic of all spines:- each has a wide and hollow core which is surrounded by a porous zone, extending to a set of radial wedges that form the majority of the solid cross-section. Spines also have a distinct pattern of microscopic barbs (Figure 1B) pointing toward the tip. Further details include bridges that link adjacent wedges as indicated in Figure 2. These bridges follow an irregular helical pattern around the longitudinal axis of the spine $[7,8]$. The central core is comprised of a thin calcite wall incorporating a regular array of holes. Overall, the spine is highly porous with an intricate structural hierarchy.

Figure $1 \mathrm{C}$ shows a scanning electron microscope (SEM) image of a fracture surface through a wedge portion of a spine which was broken by bending. The morphology of the fracture surface is similar to that observed for glassy materials $[9,10]$ rather than a cleavage-like fracture of a single crystal. The spines are strengthened due to substitution of magnesium $(\mathrm{Mg})$ for calcium $(\mathrm{Ca})$ in the carbonate crystal [5]. The $\mathrm{Mg}$ content impedes the perfect cleavage of the calcite lattice [5] in a crack-deviating mechanism, altering the fracture behavior of the calcite.

Preliminary chemical analyses were made on polished sections of spines to search for chemical variation in the materials being investigated (see Materials and Methods). This has been done as carbonate from Sea Urchin skeletons is known to vary in chemical composition $[5,6]$, partly a difference between species possibly related to temperatures of growth, partly from differences between different skeletal parts in single animals, and partly from variations across single skeletal parts (for example, $\mathrm{Mg}$ decrease from base to tip of individual spines).

Hollow cylindrical shells are a common structure in nature, for example trabecular bones, spines, quills and plant stems. This morphology, comprised of a solid outer shell with a porous core, is advantageous and effective for mechanical efficiency and high strength-to-weight ratio. Biological cylindrical structures often fail in elastic buckling due to combined axial compression and 


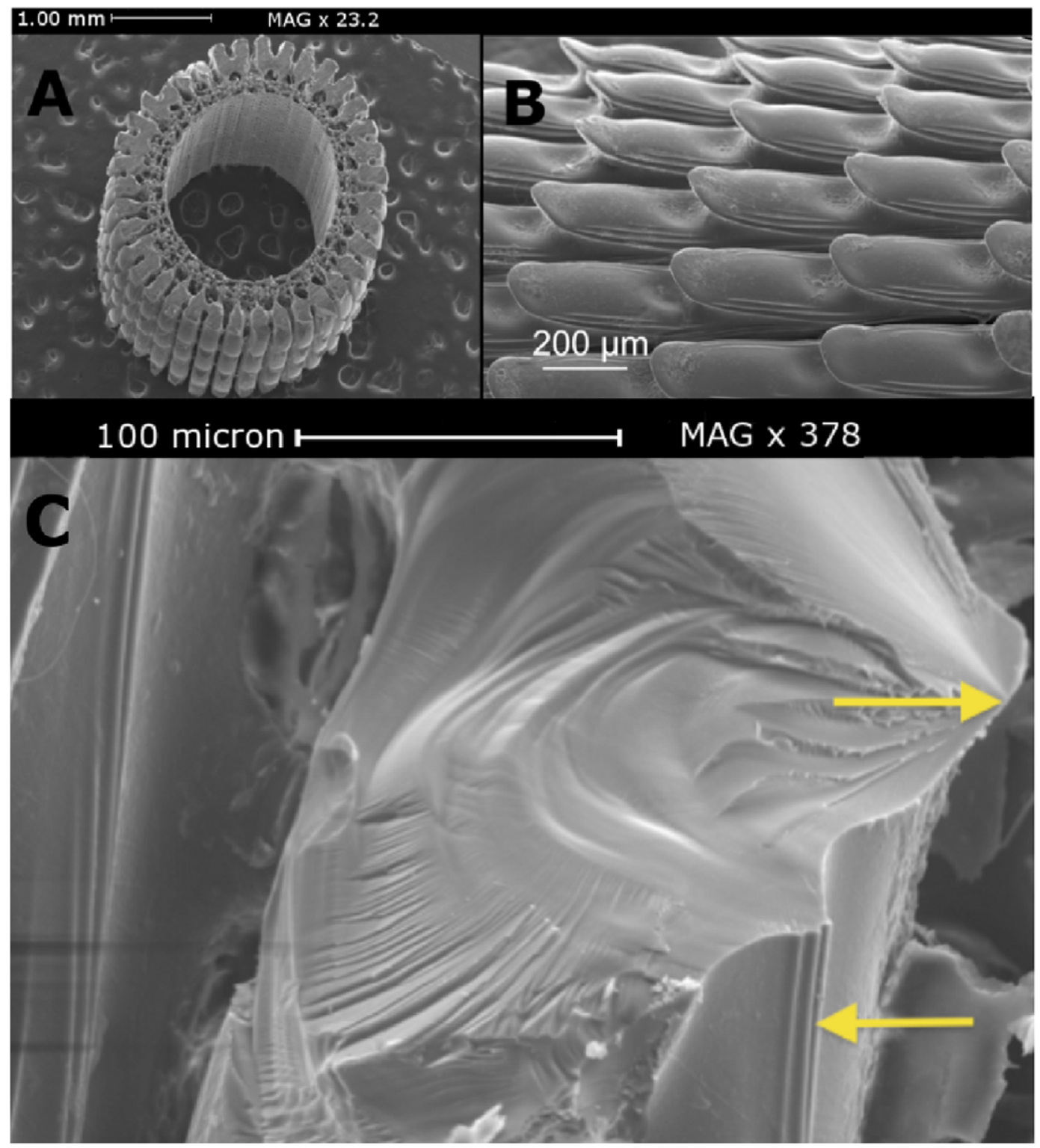

Figure 1. SEM micrographs of a Sea Urchin spine. A: Cross-section of the spine showing its hollow center and porous wall architecture (scale $\mathrm{bar}=1.0 \mathrm{~mm}$ ). B: Outer surface of the spine. Barbs point toward the spine's tip, shown here on left (scale bar $=200$ micron). C: Fracture surface of a wedge of the spine (scale bar $=100$ micron). The appearance is reminiscent of fracture morphology of glass. Top arrow points to the root of crack initiation. Bottom arrow points to a feature on the external surface of the wedge, also seen in B, which identifies the external surface, and confirms that crack initiation started on the outer surface. doi:10.1371/journal.pone.0044140.g001

bending loads [11]. However Urchin spines, with their singlecrystal material, exhibit elastic properties as well as brittle fracture.

The abaxially and radially oriented bridges spiral around the spine's axis, and together with the wedges act to concentrate mass to the outside radius of the spine [7]; it is therefore expected that in a direct collision on the spine axis, the force of impact would be transferred to the wedges, leaving the central cylinder unharmed. Spaces between wedges also serve to stop fractures from propagating through the structure, increasing the fracture strength of the spine beyond that of a monolithic calcite tube as the cracks must propagate separately in each wedge instead of propagating from one nucleation site to the entire cross-section [12].

To better understand the complexity of the spine's microstructure we created a model of a Sea Urchin spine which incorporates
3D geometry based on microCT imaging (Figure 2), and anisotropic material properties (see Materials and Methods for details). Finite element analysis was used to study a model of an urchin spine through simulated mechanical deformation. While Sea Urchin spines have been studied by microCT [8], and models using simplified geometries have been constructed to study their mechanics [12], to our knowledge this is the first study to model accurate spine microstructure based on microCT imaging. By incorporating accurate, tomography-based geometry at the micron scale into the model, the detailed contribution of each hierarchical sub-structure of the spine to the overall load-bearing capacity can be examined.

We subjected the model to compression, tension and torsion loads which the spine may encounter in nature, and studied the 


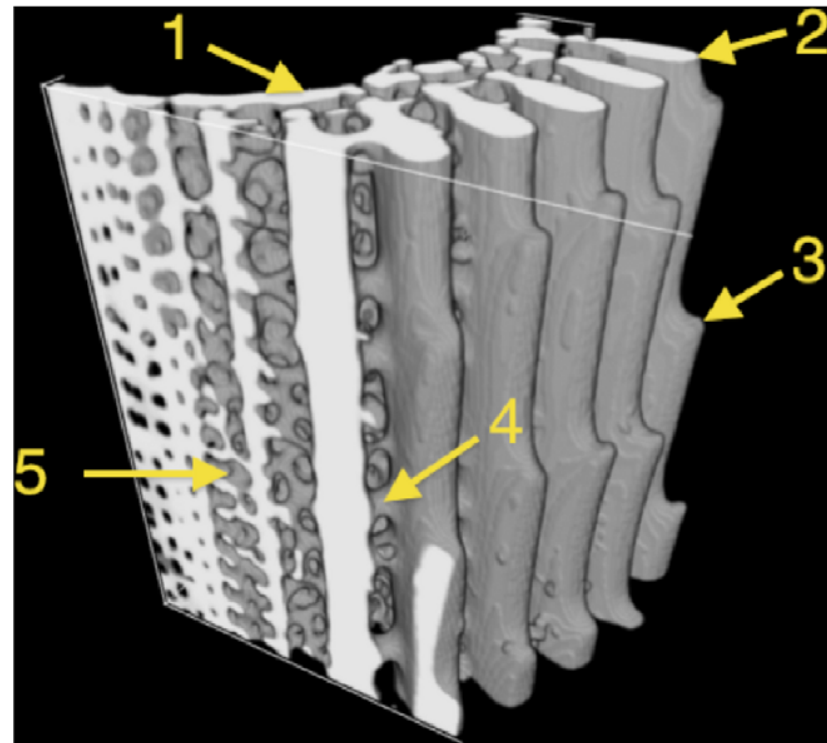

Figure 2. MicroCT reconstruction of a portion of the spine showing details of its internal anatomy. 1 - inner wall, 2 - wedge, 3 - barb, 4 - bridge, 5 - porous zone.

doi:10.1371/journal.pone.0044140.g002

resulting stress distributions. The stress and strain distributions that occur throughout the spine demonstrate how applied mechanical loads lead to different stress concentrations in the spine, resulting in either an elastically resilient structure, or one that snaps in brittle failure, depending on the type of load that is applied to it.

\section{Results and Discussion}

\section{Compression and Tension}

Compression and tension simulations gave analogous results, as expected for loads within linear elastic limits, applied along the symmetrical c-axis of the spine. Results for compression, color coded according to the level of stress, are shown in Figure 3. It can be seen that the wedges carry most of the load, and the stress in each wedge in the $x-y$ plane appears to be constant and homogeneous over the cross-sectional surface. Barbs, being protrusions from the outer surface of the wedges, are under relatively low stress. In the inner porous zone of the spine the level of stress is most heterogeneous, evidently reflecting the complexity of the microstructure.

Figure 4 shows vertical cuts through the centre of two wedges located midway in the model, far from spurious artifacts at the edges. In these images the barb profile is clearly visible, as well as the porous portion of the spine surrounding the hollow core. High stress concentration occurs in the small region of the wedge between the barbs, counterbalanced by lower stress zones extending axially into the body of the wedge. Figure 4 also shows four distinctive stress regions through the wedges: (i) a low stress region on the tip of the barb, (ii) a medium low stress region that extends further into the body of the barb and in the porous zone, (iii) a medium high stress region in the body of the wedge between barbs, and (iv) the high stress concentration already mentioned near the outer surface between barbs.

\section{Torsion}

Results of torsion loading, shown in Figure 5, provide the significant observation that there is a definite stress elevation on the bridges due to the shearing motion between the wedges as stresses applied in the xy plane cause conjugate shear stress in the yz plane. The wedges suffer relative displacement in shear along their lengths, with stress concentrating on the bridges due to their smaller cross-sectional areas. No stress is seen on the body of barbs and central cylinder. When an elastic cylinder is subjected to torsion around its longitudinal axis, the magnitude of the tangential displacement of cylinder elements in any xy crosssection is proportional to radial distance from the centre. This causes the higher stresses seen on the sides of the wedges in Figure 5, but not in the middle. In addition to the above effect, for a cylinder with trigonal symmetry, a shear strain applied in the xy plane $\left(=\varepsilon_{23}=\varepsilon_{32}\right.$ ), will cause normal strain in the $x$-axis (due to $\mathrm{c}_{24} \neq 0$ ) and $\mathrm{y}$-axis directions (due to $\mathrm{c}_{14} \neq 0$ ), but no strain in z-axis direction (due to $\mathrm{c}_{34}=0$ ). Note that the high stresses seen on the symmetry planes in Figure 5 are due to edge artifacts as boundary conditions there prevent displacements in the $\mathrm{y}$ and $\mathrm{x}$ axes.

\section{Implications of the Model}

The body of the spine is not a solid cylinder, but an assembly of wedges fanning out from the centre, interconnected by bridges. Thus, under torsion loading, the bridge appears to be the only substructure that resists the relative shearing motion of the wedges. Without these bridges the spine structure would exhibit high compliance to shearing, leading to premature structural failure. This result further supports the claim made by Stock et al [7] who discussed the significance of bridges between wedges, with wedges serving the purpose of concentrating mass to the outside radius of the spine.

The majority of high stress-concentration points in Figures 3 and 4 (modelled compressions) are situated in the wedges, especially near the barbs. These would most likely be locations where structural failure will initiate due to formation of cracks that propagate from the highly stressed surface points. Thus, the explanation of Burkhardt et al [12] in regards to the gap between wedges serving the purpose of limiting propagation of cracks seems well-founded. We have found that the wedges and the central cylinder take part in bearing stress under compressive loads. However, it is clear that the wedges act as the main support for the spine and distribute the majority of load along its body. Although bridges and barbs are attached to the wedges, they have virtually no load bearing capacity or function.

\section{Limitations of the Model}

Our simulation results were obtained for a solid of uniform composition that was chosen as a simple, first-approximation model for mechanical analysis. However minor radial composition gradients exist (see Materials and Methods) that have two direct effects. First, there will be corresponding minor gradients in the magnitudes of elastic constants, leading to small stress variations. Second, if the substitution of magnesium for calcium introduces compressive hydrostatic stress component into the outer layers of the wedge, this could lead to increased resistance to fracture by neutralizing the effect of surface micro-cracks, analogous to the classical case of fracture toughening of glass by replacing sodium with potassium ions [19].

Furthermore, we have not taken into account what effect airdrying may have on the mechanical properties of the spine. If existing, the embedded small amounts of glycoprotein should be modeled separately as soft phase inclusions in a composite material comprising a hard single-crystal matrix. This is a worthy topic of research and calls for a separate scientific study.

While this study has focused on quasi-static mechanical loads, it could be revealing to look at the effect of dynamic impact loads on 


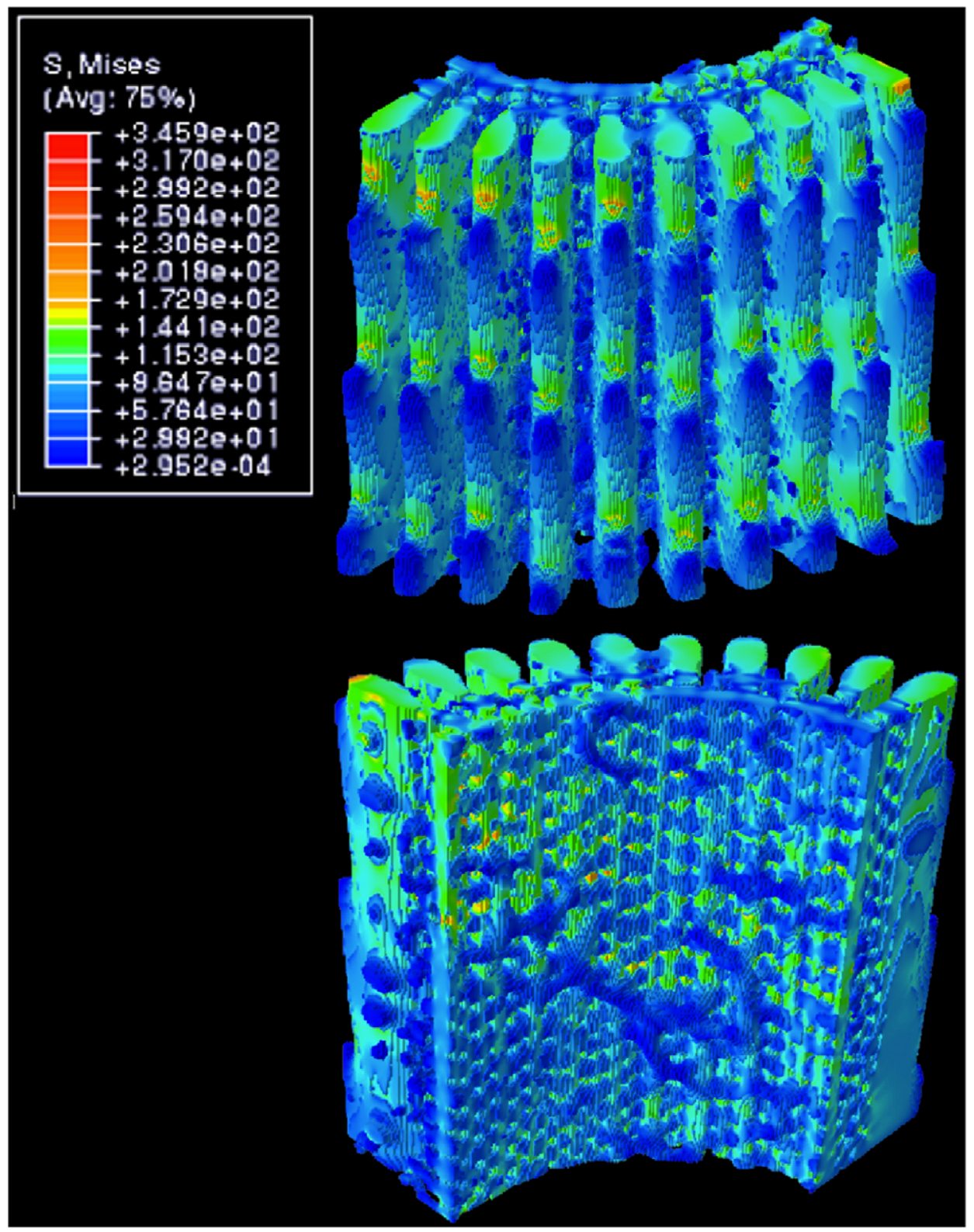

Figure 3. Distribution of von Mises stress under $1 \%$ applied compressive strain. The value of stress (MPa) is indicated in the insert; blue low level, red - high level of stress. Top: outer surface of spine. Bottom: inner surface. doi:10.1371/journal.pone.0044140.g003

spines. Further avenues of research could look at spines from several species of urchin to gauge the contribution of different morphologies to overall strength and mechanical behavior. Locomotion and sensing functions require the spines to withstand mild compressive forces with some elasticity. Indeed, the brittleness of the single-crystal calcite is tempered by the inclusion of minute amounts of organic material. When the urchin is impacted by a foreign object, the spines protect the test by absorbing the impact energy and snapping in brittle failure [1]. This requires the spines to fracture in tension under bending loads. When the spines pierce an attacking predator, they must have high longitudinal compressive strength to withstand the initial impact, but subsequently snap in brittle failure due to bending or torsion loads while remaining embedded in the predator.

\section{Conclusions}

We have characterised in detail the nature of spines in one species of Sea Urchin. This primarily involved imaging and microCT analysis of spine morphology, but also included preliminary analysis of some aspects of the chemical composition. MicroCT data was subjected to finite element analyses to investigate a range of applied load conditions, then to search for patterns of stress concentrations. We have discussed both implications and limitations of our investigations.

Bejan's constructal theory $[13,14]$ states that the optimal distribution of imperfections is a principle that underlines efficiency of form in nature, and that given time and the ability to change, systems organize themselves in a way which maximizes efficiency of flow. In the case of the Sea Urchin, the spine's structure, with its intricate barbs, wedges and bridges that act as 


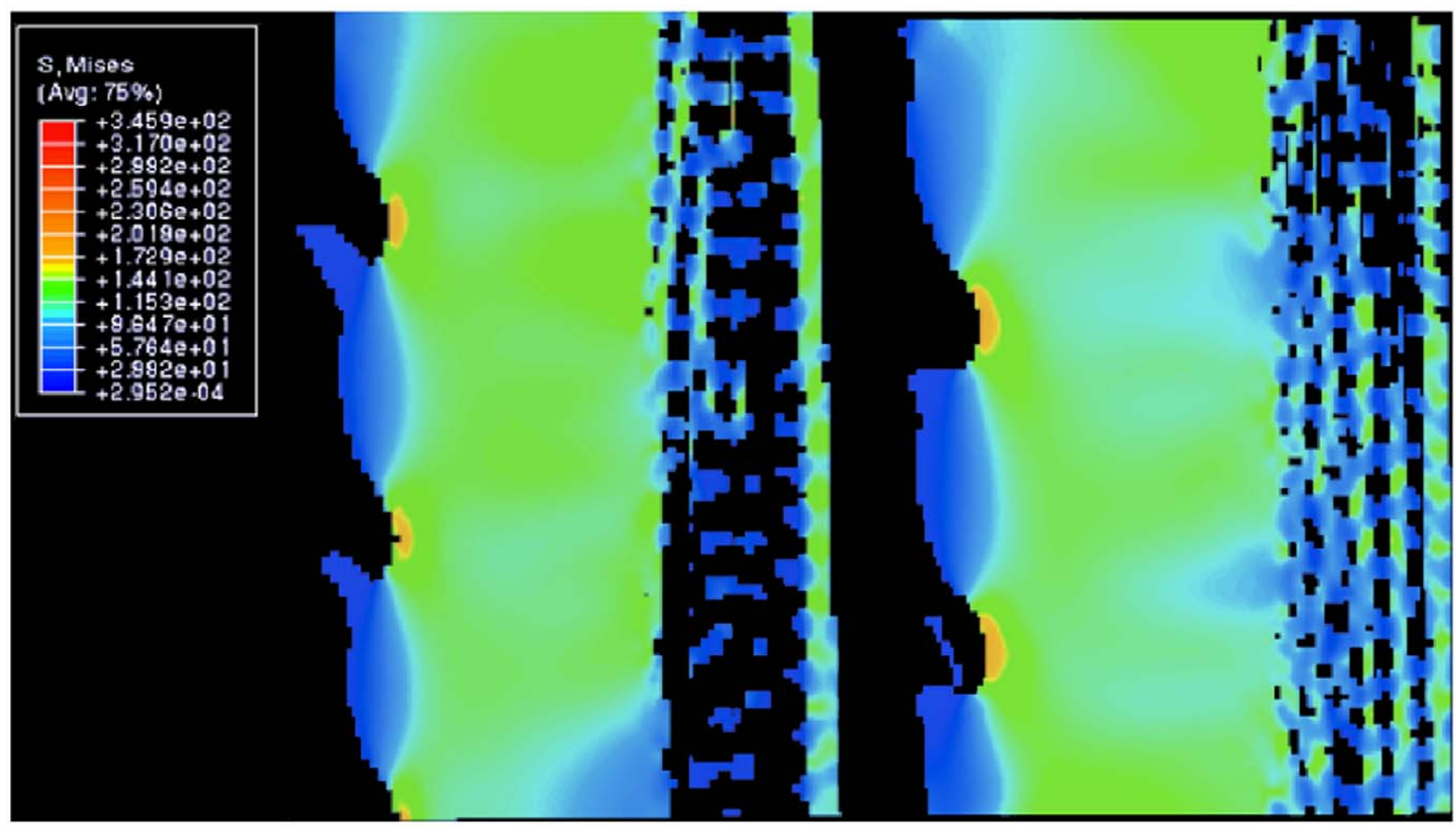

Figure 4. Vertical cuts through selected wedges showing internal stresses. The stress distributions here are cross-sectional cuts through some of the wedges shown in Figure 3, showing inhomogeneous stress, from low in the barbs (dark blue), to high in between the barbs (orange). Width of each image is approximately 600 micron. doi:10.1371/journal.pone.0044140.g004

mechanical support, contributes to its strength in bending $[7,8]$. In this case flow is not of fluid or heat, but rather of stress [15], and it seems that the urchin spine's microstructure may have evolved such that certain stress concentrations occur in response to various mechanical loadings. It is important to note that such evolutionary adaptations do not imply that the morphology is in any way ideal. However, the spine's high porosity, and the way in which its variation distributes stresses throughout its structure in response to applied loads, result in a structure that is strong and lightweight, especially considering the brittleness of the constituent material [16-18].

\section{Materials and Methods}

\section{Sample Origin}

This study focuses on the mechanical properties of the solid parts of the spines of Centrostephanus rodgersii, extracted from the Sea Urchin collected live in Batemans Bay, NSW, Australia. No specific permits were required for the described field studies. The beach where the samples were collected is public, and this species of Sea Urchin is not endangered or protected. The skeletal structure was air dried for more than two years, causing the organic tissues and membranes to naturally decay during storage in dry ambient air.

\section{MicroCT Imaging and Finite Element Analysis}

Segments of Sea Urchin spine, approximately $20 \mathrm{~mm}$ long, were scanned using microCT at a voxel resolution of 2 micron (focused electron beam, polychromatic $\mathrm{X}$-ray beam via bremsstrahlung of $80 \mathrm{kV} / 0.1 \mathrm{~mA}$, pre-filtered with a $1 \mathrm{~mm} \mathrm{CaCO}_{3}$ wafer to minimize the phenomenon of beam hardening). The samples were rotated through $360^{\circ}$ in angular increments of $0.2^{\circ}$, producing 1,800 slices of 20,482 pixels. The reconstructed three dimensional tomogram was binarized and processed using an anisotropic diffusion filter to enhance edge detection using Mango (Medial Axis and Network Generation, Australian National University and the Friedrich-Alexander-Universität ErlangenNürnberg), as seen in Figure 6.

The 3D microCT image was converted into a finite element mesh by the direct voxel conversion method; a discussion of the method is detailed elsewhere [20]. The commercial FEA suite ABAQUS (Dassault Systemes, France) was used for model preprocessing, simulation and results post-processing. Voxels were binned by a skip rate of 1 , resulting in a voxel resolution of 4 micron; reduction in resolution was necessary due to computational limits. Each voxel was converted into an 8-node hexahedral element. The spine was assumed to be an axially symmetric, cylindrical body, so one quarter of the imaged spine was modeled to keep within computational limits. Thus the highly complex, porous microstructure of the spine was modeled in the finite element mesh.

As has been shown by many microscopic, analytical and X-ray diffraction investigations (e.g. [5]) the mineral matter in the spines is $\mathrm{Mg}$-bearing rhombohedral calcite which forms one single crystal continuous throughout the complex porous solid. An invariant anisotropic stiffness tensor was therefore assigned to every solid element in the model, corresponding to the known constants for single-crystal calcite with crystallographic c-axis parallel to the Urchin spine [21].

We have verified the single-crystal nature of the spines by optical polarized microscopy and electron diffraction in TEM in our laboratory. The crystal system is trigonal, with symmetry 


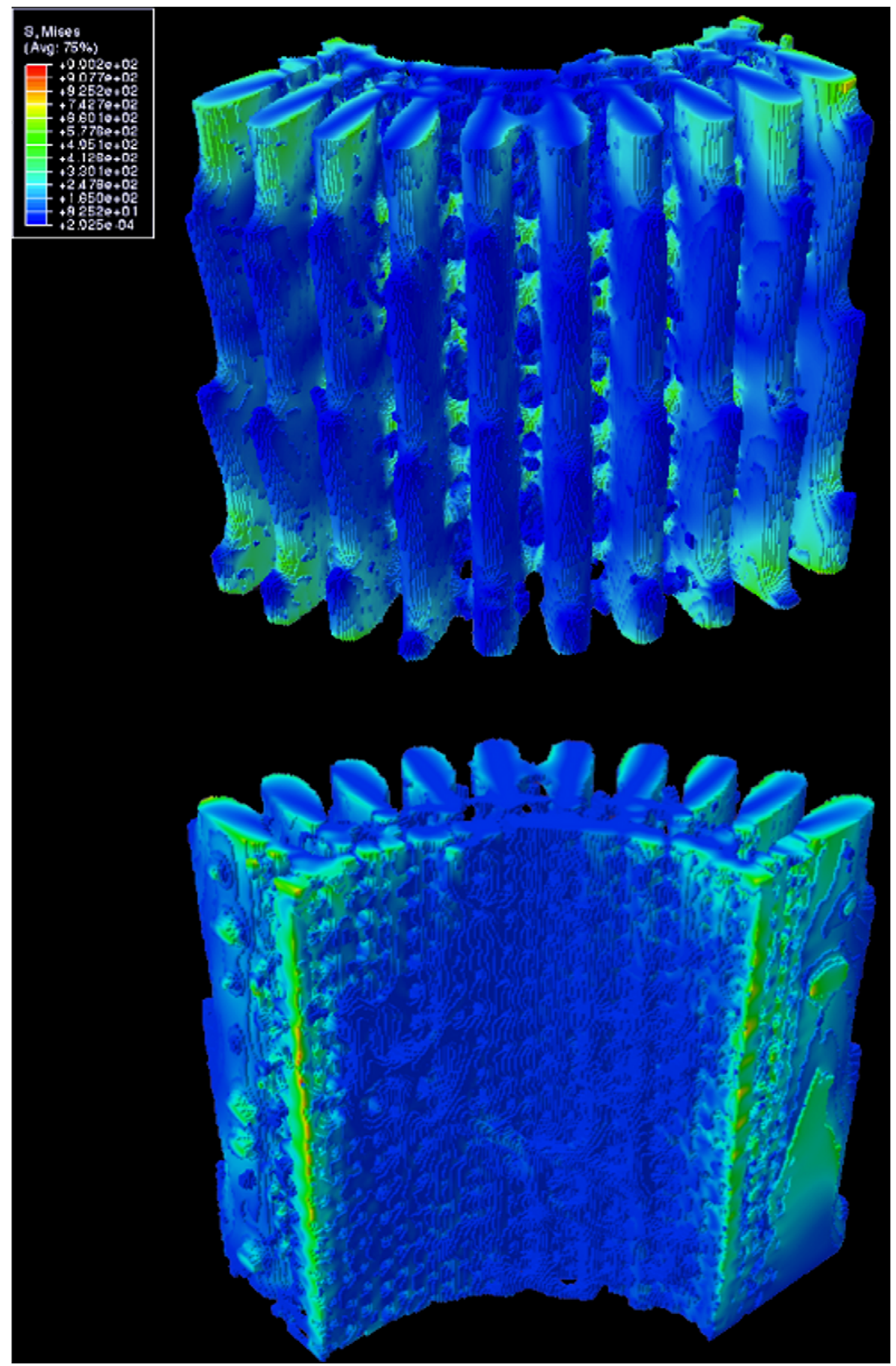


Figure 5. Distribution of von Mises stress under torsional loading. The value of stress (MPa) is indicated in the insert; blue - low level, red high level of stress. Top: outer surface of spine. Bottom: inner surface.

doi:10.1371/journal.pone.0044140.g005
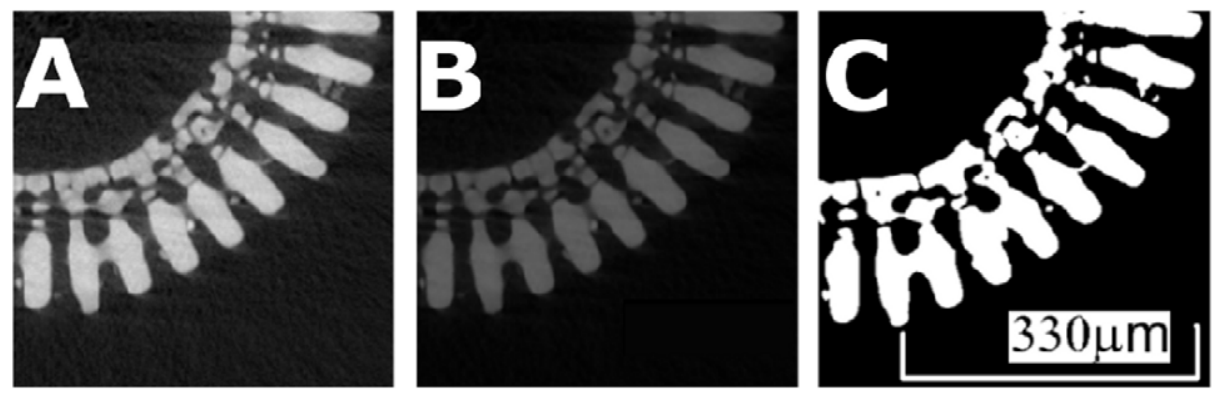

Figure 6. Contrast improvement of microCT image. A: original microCT image, B: noise reduction, and C: anisotropic diffusion filtering. doi:10.1371/journal.pone.0044140.g006

elements: $R \overline{3} c$ [22]. Since collagen (type I) also possesses trigonal symmetry [23], it would be interesting to speculate whether the trigonal crystal structures are coincidental or not. The biochemistry of these proteins is well characterized and their associated inorganic minerals are commonplace in the materials world. Politi et al. studied the transformation of amorphous calcium carbonate into calcite [24] and also reported on the mechanism of crystal formation during spine regeneration [25].

Anisotropic single-crystal properties were assigned to model elasticity in the unique structure of the crystalline spine material. The spine, with crystal [c] axis parallel to its long axis, is described by the matrix of elastic constants shown in Table 1 [21] in shorthand notation. The stress-tensor components, with generalized Hooke's law, were used to calculate the principal stresses.

Symmetry boundary conditions were set on the vertical surfaces of the model to simulate cylindrical symmetry. Three load cases were modeled by applying the following quasi-static loads to the top surface nodes: (i) $1 \%$ displacement in the $\mathrm{z}$-direction (along the spine axis) in compression, (ii) similarly in tension, and (iii) torsion modeled as a $1^{\circ}$ twist applied to the top surface around the centre axis of the cylinder.

Boundary conditions for the quarter segment were set to:

Table 1. Elastic constants for single crystal of calcite [21] used in the finite element model.

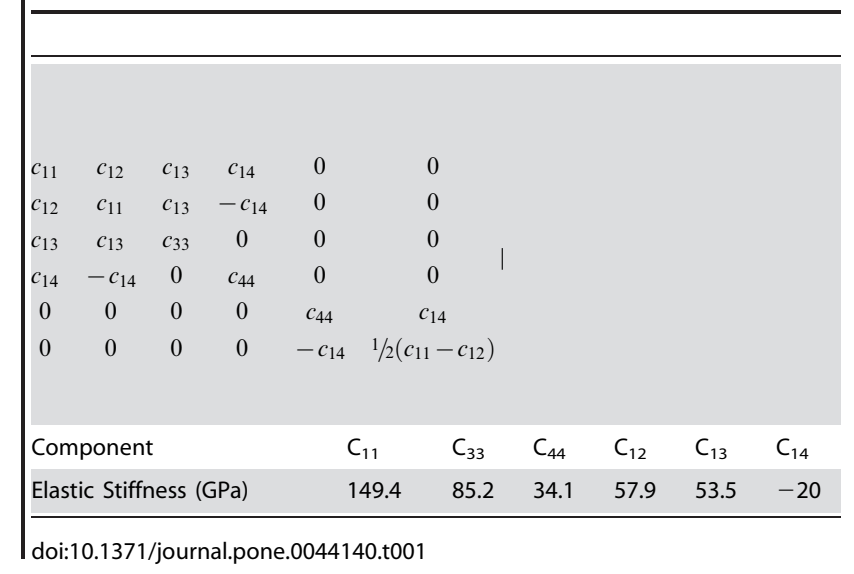

- for cases (i), (ii) and (iii) bottom surface: no rotations, no displacement in $\mathrm{z}$-axis, unrestricted displacements in $\mathrm{x}$ and $\mathrm{y}$ axis (to allow for Poisson's effect)

- for cases (i) and (ii) top surface: no rotations, unrestricted displacements in $\mathrm{x}$ - and $\mathrm{y}$-axis (to allow for Poisson's effect), $\varepsilon_{33}=-0.01$ strain for compression, or $\varepsilon_{33}=+0.01$ strain for tension

- for cases (i) and (ii) vertical $\mathrm{x}-\mathrm{z}$ surface: no rotations, no displacement in y-axis, unrestricted displacements in $\mathrm{x}$ and $\mathrm{z}$ axis

- for cases (i) and (ii) vertical $y-z$ surface: no rotations, no displacement in $\mathrm{x}$-axis, unrestricted displacements in $\mathrm{y}$ and $\mathrm{z}$ axis

- for case (iii) top surface: no rotations around $\mathrm{x}$ and $\mathrm{y}$-axes, no displacement in $\mathrm{z}$-axis direction, $1^{\circ}$ rotation around $\mathrm{z}$-axis

- for case (iii) $\mathrm{x}-\mathrm{z}$ surface: unrestricted displacements in $\mathrm{x}$ and $\mathrm{y}$ axes

- for case (iii) $y-z$ surface: unrestricted displacements in $x$ and $y$ axes

\section{Chemical Analysis}

Analyses were made using an Energy-Dispersive X-ray Spectrometer (EDS) fitted to the JEOL SEM used to image the spines. The raw data could be roughly assessed by inspecting the EDS spectra, but for better reliability over 50 chemical analyses were made from micron-sized regions, each analysis being processed from spectra via the procedures known in general as ZAF correction [26] using verified standards. In addition, some uncorrected chemical maps were made to reveal spatial distribution of X-ray intensities, a technique widely used to indicate chemical variation.

In longitudinal sections, no chemical variability from spine base to tip could be discerned. However, variability was recorded in a transverse section. Figure 7 shows a micrograph of this specimen recorded using SEM. The area used for X-ray mapping is indicated by the rectangle marked and detailed in Figure 8. X-ray maps with higher intensity indicating increased chemical abundance of the corresponding element, are shown for $\mathrm{Mg}$ and $\mathrm{S}$ in Figure 9. The variation extends across the spine wedge. Other elements measured do show variations not inside the wedge but restricted to the hard-spine surfaces and dismissed as related to surface coatings or contaminations. 


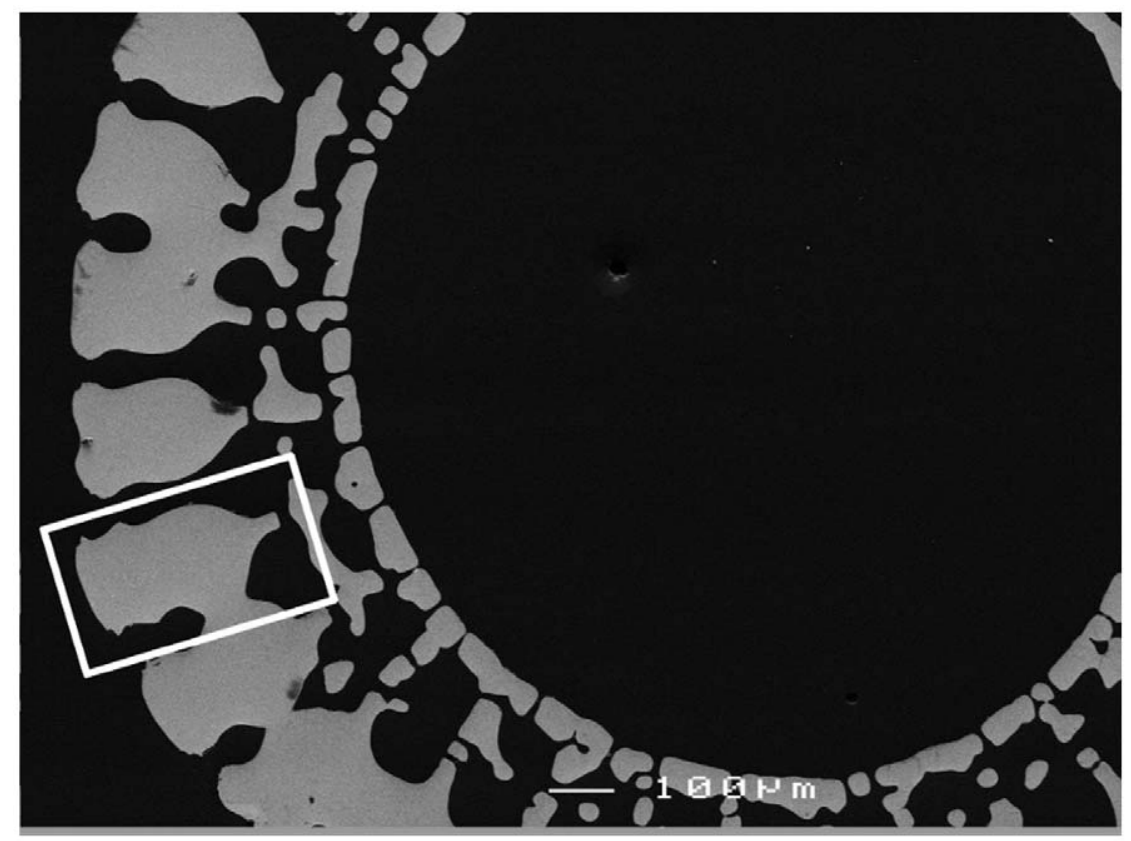

Figure 7. SEM micrograph of spine transverse section. White rectangle indicates single wedge area used for chemical investigation. doi:10.1371/journal.pone.0044140.g007

To further characterize chemical variation, corrected analyses were made along a line from the tip to the base of the same wedge (see ten analysis marks in Figure 8). In general the analyses confirm the trends revealed from mapping, for example while the composition of the carbonate is mainly $4 \%-5 \%$ molar $\mathrm{MgCO}_{3}$, it appears to vary from approx $2.55 \%$ to $6 \%$ along the line analyzed. Plots of $\mathrm{Mg}, \mathrm{S}, \mathrm{Ca}$ and $\mathrm{Mg} /(\mathrm{Mg}+\mathrm{Ca})$ are shown in Figure 10. There is clearly a decrease in $\mathrm{Mg}$ towards the wedge tip. There is also a clear increase in $\mathrm{S}$ at the wedge surface, but both maps and profiles suggest this is spatially restricted compared to the $\mathrm{Mg}$ variation.

These chemical analyses indicate variation in composition of the carbonate with some trends identified for one area in detail. However, much more careful analytical work is required to definitely establish the patterns and examine whether they apply to all wedges and spines. Further analyses along the spine length are also warranted.

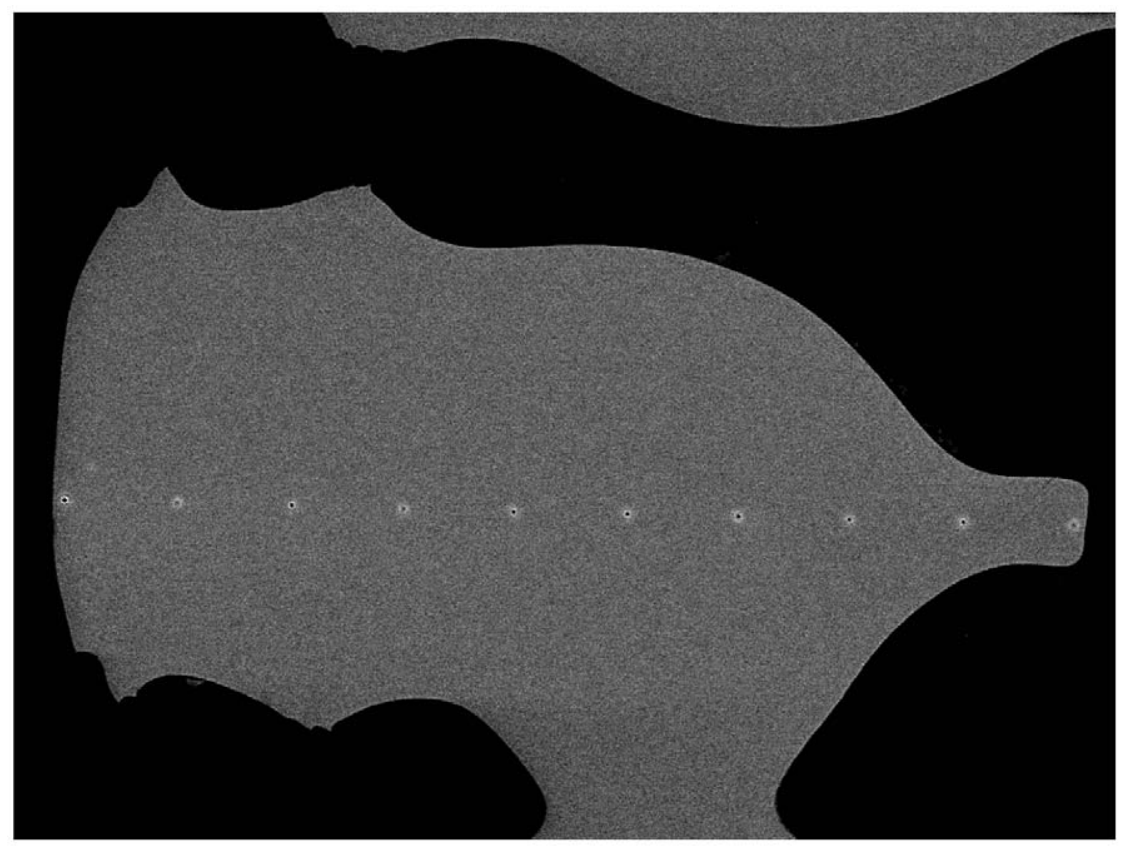

Figure 8. SEM micrograph of the area after chemical analysis. Note the line of 10 analysis spots at a spacing of 38 micrometers along a line from the tip to the base of this wedge. doi:10.1371/journal.pone.0044140.g008 

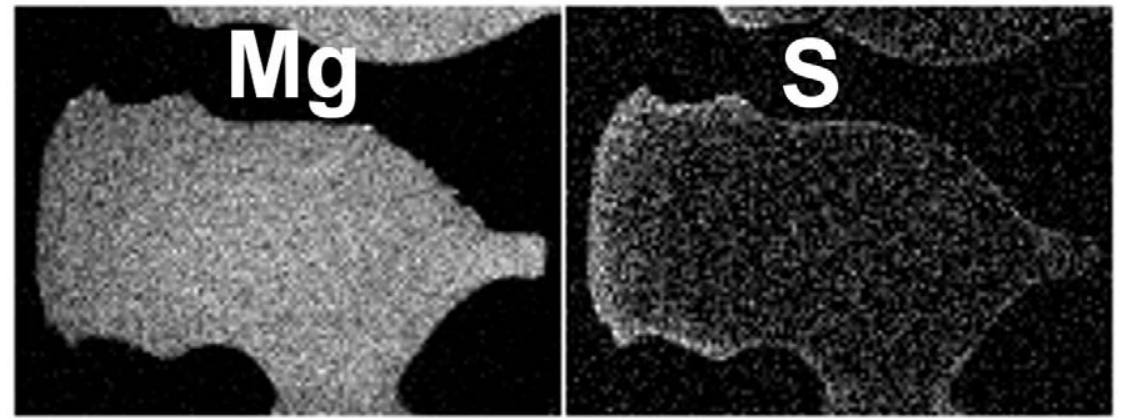

Figure 9. Maps of X-ray intensities. Intensities of $\mathrm{Mg}$ (left) and S (right) detected across the area used for chemical analyses. In both maps, brightest regions are those with highest abundance.

doi:10.1371/journal.pone.0044140.g009

$100 \mathrm{Mg} /(\mathrm{Mg}+\mathrm{Ca})$

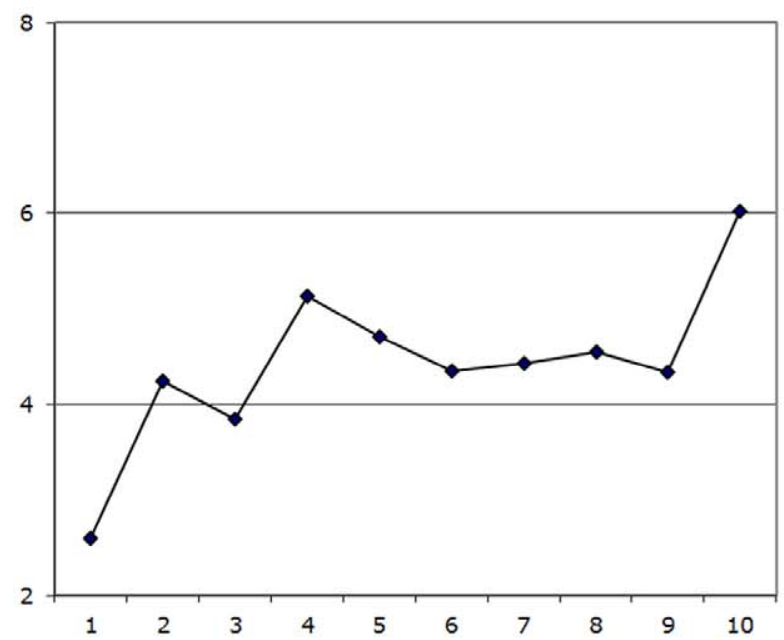

Wt $\%$ equiv $\mathrm{SO}_{2}$

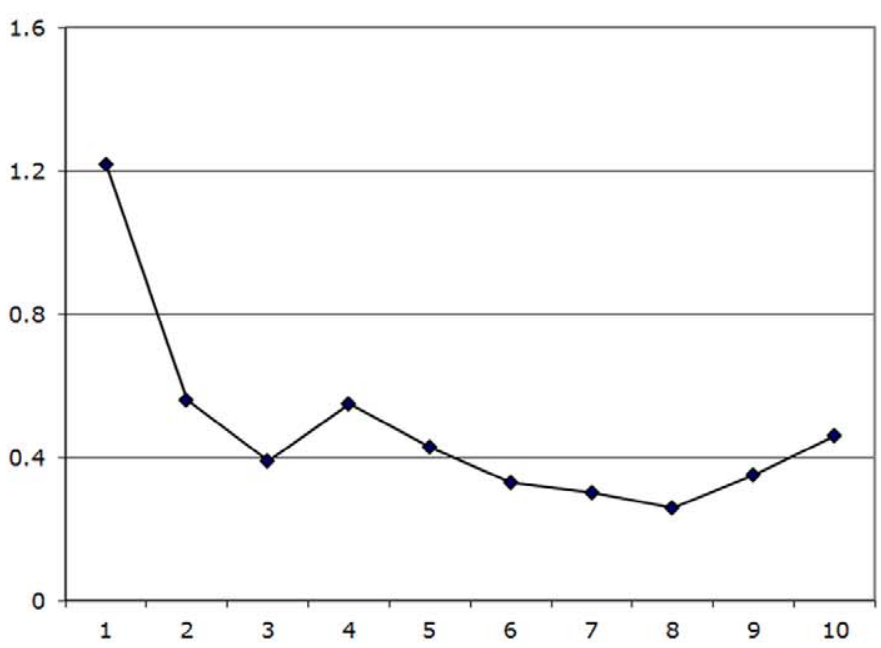

Figure 10. Chemical analyses. Representative results of analyses made along the line indicated in Figure 8. Some variation in composition was measured from the wedge tip (point 1 plotted at left) to the base (point 10 plotted at right), reinforcing the trends from X-ray-intensity maps of Figure 9.

doi:10.1371/journal.pone.0044140.g010

\section{Acknowledgments}

We wish to acknowledge help from staff of the ANU Supercomputing Facility, Dept. of Applied Mathematics (M. Turner and T. Senden, RSPE), specimen preparation by (Harri Kokkonen, RSES), and ANU CAM staff for SEM and microanalysis. The authors are grateful for scientific and technical input and support from the Australian Microscopy and Microanalysis Research Facility (AMMRF) node at the University of Sydney and ANU. AnYao Liu carried out preliminary optical and electron

\section{References}

1. Strathmann RR (1981) The Role of Spines in Preventing Structural Damage to Echinoid Tests. Paleobiology 7: 400-406.

2. Donovan SK (1999) Functional Morphology Of The Invertebrate Skeleton. In: Savazzi E, editor: John Wiley and Sons. 565-600.

3. Berman A, Addadi L, Kvick Å, Leiserowitz L, Nelson M, et al. (1990) Intercalation of Sea Urchin Proteins in Calcite: Study of a Crystalline Composite Material. Science 250: 664-667.

4. Stupp SI, Braun PV (1997) Molecular Manipulation of Microstructures: Biomaterials, Ceramics, and Semiconductors. Science 277: 1242-1248.

5. Magdans U, Gies H (2004) Single crystal structure analysis of sea urchin spine calcites: Systematic investigations of the $\mathrm{Ca} / \mathrm{Mg}$ distribution as a function of microscopic studies. I. Jackson collected the live urchins which were used for this study.

\section{Author Contributions}

Conceived and designed the experiments: NT JDFG ZHS. Performed the experiments: NT JDFG HNL ZHS. Analyzed the data: NT JDFG HNL ZHS. Contributed reagents/materials/analysis tools: NT JDFG ZHS. Wrote the paper: NT JDFG HNL ZHS. Obtained microCT images: HNL. Carried out finite element analysis: NT HNL. Supervised the study: JDFG ZHS.

habitat of the sea urchin and the sample location in the spine. European Journal of Mineralogy: 261-268.

6. Seto J, Ma Y, Davis S, Meldrum F, Gourrier A, et al. (2012) Structure-property relationships of a biological mesocrystal in the adult sea urchin spine. Proceedings of the National Academy of Sciences 109: 3699-3704.

7. Stock SR, Carlo F, Ebert TA (2009) Bridges between radial wedges (septs) in two diadematid spine types. Echinoderms: Durham: CRC Press. 263-267.

8. Stock SR, Ebert TA, Ignatiev K, De Carlo F (2006) Structures, structural hierarchy and function in sea urchin spines. Developments in X-Ray Tomography V. San Diego, CA. 
9. Lawn B (1993) Fracture of Brittle Solids (Cambridge Solid State Science Series): Cambridge University Press.

10. Woodward A (1989) Atlas of Polymer Morphology: Hanser Gardner Pubns.

11. Karam GN, Gibson LJ (1994) Biomimicking of animal quills and plant stems: natural cylindrical shells with foam cores. Materials Science and Engineering: $\mathrm{C}$ 2: 113-132.

12. Burkhardt A, Hansmann W, Märkel K, Niemann H-J (1983) Mechanical design in spines of diadematoid echinoids (Echinodermata, Echinoidea). Zoomorphology 102: 189-203.

13. Bejan A (2000) Shape and Structure, from Engineering to Nature: Cambridge University Press.

14. Bejan A, Lorente S (2010) The constructal law of design and evolution in nature. Philosophical Transactions of the Royal Society B: Biological Sciences 365: 1335-1347.

15. Lorente S, Lee J, Bejan A (2010) The "flow of stresses" concept: The analogy between mechanical strength and heat convection. International Journal of Heat and Mass Transfer 53: 2963-2968.

16. Abou Chakra M, Stone JR (2011) Holotestoid: A computational model for testing hypotheses about echinoid skeleton form and growth. Journal of Theoretical Biology 285: 113-125.

17. Abou Chakra M, Stone J (2011) Classifying echinoid skeleton models: testing ideas about growth and form. Paleobiology 37: 686-695.
18. Zachos LG (2009) A new computational growth model for sea urchin skeletons. Journal of Theoretical Biology 259: 646-657.

19. Sugarman B (1967) Strength of glass (a review). Journal of Materials Science 2: 275-283.

20. Tsafnat N, Tsafnat G, Jones AS (2008) Micro-finite element modelling of coke blends using X-ray microtomography. Fuel 87: 2983-2987.

21. Chen C-C, Lin C-C, Liu L-G, Sinogeikin S, Bass J (2001) Elasticity of singlecrystal calcite and rhodochrosite by Brillouin spectroscopy. American Mineralogist 86: 1525-1529.

22. Newnham R (2005) Properties of Materials: Anisotropy, Symmetry, Structure: Oxford University Press, USA.

23. Jiang B-Y, Chu S-W (2008) Trigonal symmetry of type I collagen probed by SHG polarization anisotropy. Lasers and Electro-Optics, 2008 and 2008 Conference on Quantum Electronics and Laser Science CLEO/QELS 2008 Conference on: $1-2$.

24. Politi Y, Metzler R, Abrecht M, Gilbert B, Wilt F, et al. (2008) Transformation mechanism of amorphous calcium carbonate into calcite in the sea urchin larval spicule. Proceedings of the National Academy of Sciences 105: 17362-17366.

25. Politi Y, Arad T, Klein E, Weiner S, Addadi L (2004) Sea Urchin Spine Calcite Forms via a Transient Amorphous Calcium Carbonate Phase. Science 306: 1161-1164.

26. Russ JC (1984) Fundamentals of energy dispersive x-ray analysis: Butterworths. 